\title{
The added value of pirfenidone to declare war on inflammation and the fibrotic state induced by Sars Cov 2
}

\author{
Francesco Ferrara ${ }^{1}$, Giovanni Granata ${ }^{2}$, Chiara Pelliccia ${ }^{3}$, Raffaele La Porta ${ }^{4}$, and Antonio \\ Vitiello ${ }^{1}$ \\ ${ }^{1}$ Azienda Unità Sanitaria Locale Umbria 1 \\ ${ }^{2}$ Local Health Authority Salerno \\ ${ }^{3}$ Azienda Unità Sanitaria Locale Umbria 2 \\ ${ }^{4}$ ASUR Marche
}

April 27, 2020

\begin{abstract}
Reduction of pulmonary fibrotic status and reduction of hyperinflammation is essential to combat SARS-CoV-2 and avoid death. Many authors have divided the SARS-CoV-2 infection into three stages, the second and third of which are purely inflammatory and fibrotic. Waiting for the development of antiviral drugs and vaccines to give good results, the best pharmacological goal is the reduction of proinflammatory molecules. This leads to less formation of fibrotic tissue and to the resolution of the patient's respiratory problems. In fact, in phase 3, the most serious, there is a state of overexpression of the immune system with consequent assault on all tissues and damage to the lungs. Sars cov 2 pneumonia is characterized by "cytokine storm" and can lead to death. Acting early and with pirfenidone combination therapy can be effective. The IL-6 or IL-1 inhibitors, chloroquine / hydroxychloroquine and colchicine, which are demonstrating their anti-inflammatory efficacy, when combined with an antiinflammatory and antifibrotic agent, such as pirfenidone, can have a winning result. The effective combined terepia allows to use non-lethal dosages and affects all the pathological steps induced by the virus. Pirfenidone has been used for years in lung diseases and has been shown to have good clinical success and good safety and tolerability. The purpose of this study is to explain the pharmacological logic behind the use of a combination therapy as an effective and safe remedy to reduce pneumonia and the consequent death from Sars CoV 2. Keywords: pirfenidone, fibrotic, inflammation, cythokine, interleukin, Sars-CoV-2.
\end{abstract}

The added value of pirfenidone to declare war on inflammation and the fibrotic state induced by Sars Cov 2

Anti-inflammatory and antiviral therapy with the addition of pirfenidone can resolve fibrotic and cicatricial complications of the lung

Francesco Ferrara 1*, Giovanni Granata 2, Chiara Pelliccia 3, Raffaele La Porta PhD 4, Antonio Vitiello 1

1: Usl Umbria 1, Perugia, Italy

2: Asl Salerno, Salerno, Italy

3: Usl Umbria 2, Terni, Italy

4: Asur Marche, Ancona, Italy

*author correspondence: francesco.ferrara@uslumbria1.it 


\begin{abstract}
Reduction of pulmonary fibrotic status and reduction of hyperinflammation is essential to combat SARSCoV-2 and avoid death. Many authors have divided the SARS-CoV-2 infection into three stages, the second and third of which are purely inflammatory and fibrotic. Waiting for the development of antiviral drugs and vaccines to give good results, the best pharmacological goal is the reduction of proinflammatory molecules. This leads to less formation of fibrotic tissue and to the resolution of the patient's respiratory problems. In fact, in phase 3, the most serious, there is a state of overexpression of the immune system with consequent assault on all tissues and damage to the lungs. Sars cov 2 pneumonia is characterized by "cytokine storm" and can lead to death. Acting early and with pirfenidone combination therapy can be effective. The IL-6 or IL-1 inhibitors, chloroquine / hydroxychloroquine and colchicine, which are demonstrating their antiinflammatory efficacy, when combined with an anti-inflammatory and antifibrotic agent, such as pirfenidone, can have a winning result. The effective combined terepia allows to use non-lethal dosages and affects all the pathological steps induced by the virus. Pirfenidone has been used for years in lung diseases and has been shown to have good clinical success and good safety and tolerability.The purpose of this study is to explain the pharmacological logic behind the use of a combination therapy as an effective and safe remedy to reduce pneumonia and the consequent death from Sars CoV 2.
\end{abstract}

Keywords : pirfenidone, fibrotic, inflammation, cythokine, interleukin, Sars-CoV-2.

\title{
Introduction
}

Sars-CoV-2 infection leads to hyperinflammation, fibrotic and cicatricial state, lung collapse and death of the patient if he is at risk. This clinical picture must be contrasted to put an end to a global pandemic that has already caused thousands of victims and this number is expected to grow exponentially. If the health crisis that has hit the whole world lasts for a long time, it risks generating a deep economic crisis with all the activities stopped and the continuous lock dawn imposed. For these reasons, looking for a therapy that can make the virus harmless immediately is of fundamental importance. Avoiding death is a great success that allows you to have the time to find a drug directed against the virus without blocking the whole world. Preventing patient death is a success to be achieved. The excessive inflammatory state induced by the Sars-CoV-2 virus has been shown to aggravate the patient's clinical picture. In the most serious phases of the pathology there is an excessive release of proinflammatory cytokines that lead to lung damage with extensive fibrosis and scarring of the interstitial lungs with respiratory impairment. The combined use of anti-inflammatory drugs and drugs that prevent fibrotic status can beneficially resolve the clinical picture. Today Pirfenidone is the only drug that has been shown to reduce the fibrotic state of the lung tissues and is the drug of choice in many lung diseases. Combination therapy with pirfenidone can lead, albeit indirectly, to the defeat of the virus.

\section{The clinical aspects of three phases SARS-CoV-2 infection}

The SARS-Cov-2 virus caused the current global pandemic. The first cases of SARS-Cov-2 infection were recorded in November 2019 in China and then rapidly spread to all countries around the world causing thousands of deaths. SARS-Cov-2 infection can rapidly cause respiratory distress syndrome. According to observational studies conducted, the majority of patients considered as severe cases present bilateral intestinal pneumonia, and an overactive inflammatory state that is not only localized to lung tissue but to all tissues of the body. SARS-Cov-2 infection has been divided by scientific opinion into three phases, the first asymptomatic or mildly symptomatic with symptoms such as mild sore throat and abdominal pain, phase two and three more severe than one, presenting with a generalized inflammatory state and respiratory distress syndrome. Evidence has shown that bilateral interstitial pneumonia, defined in this way because it attacks the intestinal tissue covering the lung alveoli, is associated with the presence of fibrotic tissue caused by excess collagen (fibrosis) in the pulmonary interstitium with hyperinflammation present. Several therapeutic treatments have been considered, and several clinical trials are underway to test their efficacy and 
safety, drugs such as antivirals, immunomodulants, anticoagulants are being tested to best fight SARS-Cov-2 infection. As described above, phase two and three are the most serious, and the presence of inflammation in the lungs and scar tissue and fibrotic tissue, requires immunomodulating and anti-inflammatory treatment to combat these phases, although at the moment there are no certain data that demonstrate their effectiveness and at what time they should be used, ongoing clinical trials will give us more evidence. However, based on the descriptions of symptomatology and diagnostic investigations from early observational studies, one might think that the use of a drug currently indicated for pulmonary fibrosis such as pyrfenidone could bring great benefits. We also consider that given the very complex nature of the pathophysiology of SARS-Cov-2, probably a combination of several drugs (pending the marketing of a direct and specific antiviral or vaccine) in several steps could be the most suitable treatment (1-23).

\section{Pirfenidone}

Pirfenidone is administered orally 3 times a day, 2 or 3 tablets each time, for a period of 4 weeks or longer. The mechanism of action of pyrfenidone has not yet been fully determined. However, existing data indicate that pyrfenidone exerts both antifibrotic and anti-inflammatory properties in a variety of in vitro systems and animal models of pulmonary fibrosis (bleomycin-induced and transplant fibrosis). IPF is a chronic fibrotic and inflammatory pulmonary disease caused by the synthesis and release of pro-inflammatory cytokines including tumor necrosis factor alpha $(\mathrm{TNF}-\alpha)$ and interleukin-1-beta (IL-1 $\beta$ ), and pyrfenidone has been shown to reduce the accumulation of inflammatory cells in response to various stimuli. Pirfenidone attenuates fibroblast proliferation, protein and cytokine production associated with fibrosis and increased biosynthesis and extracellular matrix accumulation in response to cytokine growth factors such as transformative growth factor beta (TGF- $\beta$ ) and platelet-derived growth factor (PDGF) (Figure 1 ).

The acute lung injury caused by SARS and 2003 were both related to the inflammatory cytokine storm in patients. The biochemical test showed abnormal increase in related indicators such as interleukin-8, and CT images showed a medical "white" lung". According to the experience of SARS treatment in 2003, the use of hormones will indeed help the patients to alleviate their illness, but patients who survived SARS either had too much hormone at that time and took too long. Although the lungs could recover, but the femoral head was necrotic Either the amount of hormones was very conservative at the time, which kept the lungs in the storm of inflammatory factors, leading to the emergence of irreversible pulmonary fibrosis. So is there a medicine that can anti-inflammatory, reduce the load of hormone use, and have the effect of treating and preventing pulmonary fibrosis complicated by severe viral lung? At present, pirfenidone has achieved encouraging results in the treatment of idiopathic Pulmonary Fibrosis (CTD-ILD) diseases. It is particularly encouraging that the values announced at the 2019 ATS Annual Conference suggest that pirfenidone has more anti-inflammatory and anti-oxidant effects than its own outstanding anti-fibrotic ability. The data shows early use, Its strong anti-SOD activity can effectively inhibit IL-1beta and IL-4, and can open the prevention mode of pulmonary interstitial fibrosis. Based on the above, this project intends to make the following scientific assumptions: based on the homology of the pathogens of the new coronavirus-infected pneumonia and the coronavirus infection of pneumonia in 2003, the similarities in the occurrence and development of the disease, that is, the pulmonary inflammatory storm occurs first, and thereafter The progress of fibrosis and the progressive decline of lung function and mortality are higher than those of ordinary pneumonia. We hope that by adding pirfenidone as a treatment program in addition to standard treatment, it will be a new and severe type of coronavirus infection. Patient clinical treatment provides an effective and practical method (24-26).

A trial is also starting recently which will give strength to our hypothesis, "A Study to Evaluate the Efficacy and Safety of Pirfenidone With Novel Coronavirus Infection", conducted in Wuhan, China. 


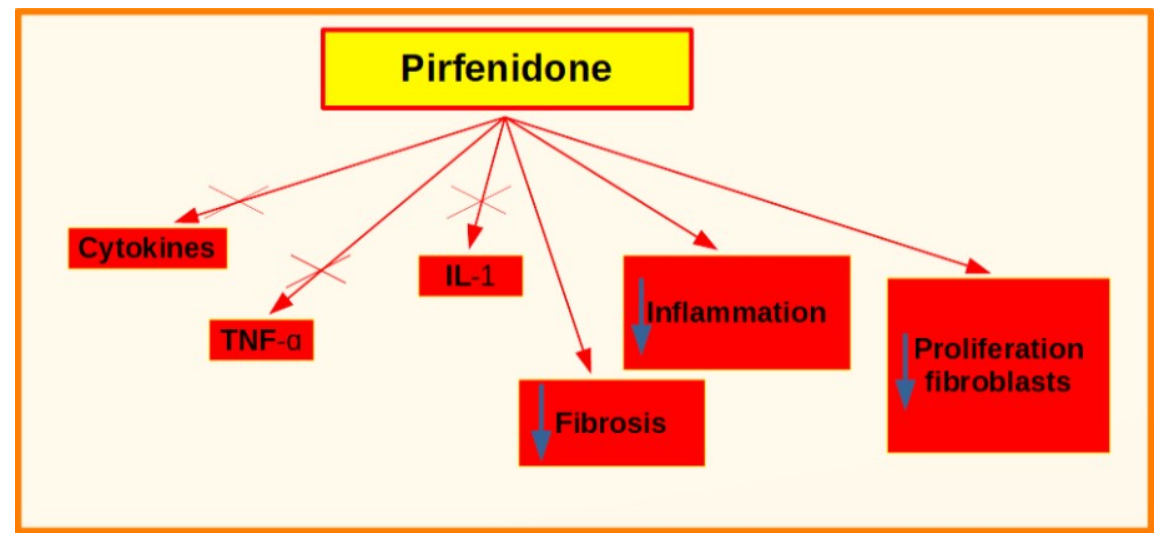

Figure 1 : Mechanisms of action of pirfenidone.

\section{Conclusions}

The severe inflammatory state caused by Sars-CoV-2 infection leads to lung collapse, respiratory difficulties due to fibrosis and death of the patient. The sudden release of cytokines in the circulation, known as "cytokine storm" (CS), is a phenomenon that must be countered in order not to get scarring of the lung tissue. To date, there are still no effective antivirals that can prevent the evolution of this clinical picture and, pending better solutions, it is good to avoid the patient's death with the blocking of inflammation and the dissolution of the fibrotic tissue formed. The goal is to save the patient's life. Correct management of the inflammatory / immune status of the infected patient takes on a priority role. In the third stage, the more serious one, the combined use of anti-inflammatory drugs and pirfenidone can help avoid death from SARS-CoV-2. The use of pirfenidone, for its good tolerability and safety, could be a winning move. In addition, the combined use of multiple drugs allows a safe and non-risky dosage compared to monotherapy and is certainly the most effective and tolerable solution for managing the inflammatory and fibrotic state.

\section{MAIN STATEMENTS}

I, the undersigned, Francesco Ferrara and any other author, declare that:

We have no conflict of interest; We have not received funding; There are no sensitive data and no patients were recruited for this study; The document does not conflict with ethical legislation.

Regards

The authors

\section{References}

1. Li YC Bai \& WZ Hashikawa T. The neuroinvasive potential of SARS-CoV2 may play a role in the respiratory failure of COVID-19 patients.J Med Virol. 2020 Feb 27. doi: 10.1002/jmv.25728.

2. Glass WG, Subbarao K, Murphy B, Murphy PM. Mechanisms of host defense following severe acute respiratory syndrome-coronavirus (SARS-CoV) pulmonary infection of mice. J Immunol . 2004; 173: 4030- 4039.

3. Zhao W, Zhong Z, Xie X, Yu Q, Liu J Relation Between Chest CT Findings and Clinical Conditions of Coronavirus Disease (COVID-19) Pneumonia: A Multicenter Study. AJR Am J Roentgenol. 2020 Mar 3:1-6. doi: 10.2214/AJR.20.22976.

4. Huang C, Wang Y, Li X, et al. Clinical features of patients infected with 2019 novel coronavirus in Wuhan, China. Lancet. 2020; 395: 497- 506. 
5. Yu F, Du L, Ojcius DM, Pan C, Jiang S. Measures for diagnosing and treating infections by a novel coronavirus responsible for a pneumonia outbreak originating in Wuhan, China. Microbes Infect. 2020. https://doi.org/10.1016/j.micinf.2020.01.003.

6. Song Z, Xu Y, Bao L, et al. From SARS to MERS, thrusting coronaviruses into the spotlight. Viruses. 2019; 11: 59. https://doi.org/10.3390/v11010059

7. Lu R, Zhao X, Li J, et al. Genomic characterisation and epidemiology of 2019 novel coronavirus: implications for virus origins and receptor binding. Lancet. 2020; 395: 565- 574.

8. Wan Y, Shang J, Graham R, Baric RS, Li F. Receptor recognition by novel coronavirus from Wuhan: an analysis based on decade-long structural studies of SARS. J Virol . 2020. https://doi.org/10.1128/JVI.0012720

9. Yuan Y, Cao D, Zhang Y, et al. Cryo-EM structures of MERS-CoV and SARS-CoV spike glycoproteins reveal the dynamic receptor binding domains. Nat Commun. 2017; 8:15092.

10. Hulswit RJ, de Haan CA, Bosch BJ. Coronavirus spike protein and tropism changes. Adv Virus Res. 2016; 96: 29- 57.

11. Li YC, Bai WZ, Hirano N, Hayashida T, Hashikawa T. Coronavirus infection of rat dorsal root ganglia: ultrastructural characterization of viral replication, transfer, and the early response of satellite cells. Virus Res. 2012; 163: 628- 635.

12. Wang D, Hu B, Hu C, et al. Clinical characteristics of 138 hospitalized patients with 2019 novel coronavirus-infected pneumonia in Wuhan, China. JAMA . 2020.

13. Khan S, Ali A, Siddique R, Nabi G. Novel coronavirus is putting the whole world on alert. J Hosp Infect. 2020. https://doi.org/10.1016/j.jhin.2020.01.019

14. Chen N, Zhou M, Dong X, et al. Epidemiological and clinical characteristics of 99 cases of 2019 novel coronavirus pneumonia in Wuhan, China: a descriptive study. Lancet. 2020; 395(10223): 507- 513.

15. Li K, Wohlford-Lenane C, Perlman S, et al. Middle East respiratory syndrome coronavirus causes multiple organ damage and lethal disease in mice transgenic for human dipeptidyl peptidase 4. J Infect Dis. 2016; 213: 712- 722 .

16. Talbot PJ, Ekandé S, Cashman NR, Mounir S, Stewart JN. Neurotropism of human coronavirus 229E. Adv Exp Med Biol . 1993; 342: 339- 346.

17. Dube M, Le Coupanec A, Wong AHM, Rini JM, Desforges M, Talbot PJ.Axonal transport enables neuron-to-neuron propagation of human coronavirus OC43. J Virol. 2018; 92, https://doi.org/10.1128/JVI.0040418

18. Zhou X, Huang F, Xu L, et al. Hepatitis E virus infects neurons and brains. J Infect Dis. 2017; 215(8): $1197-1206$.

19. Li YC, Bai WZ, Hirano N, et al. Neurotropic virus tracing suggests a membranous-coating-mediated mechanism for transsynaptic communication. J Comp Neurol. 2013; 521: 203- 212.

20. Mengeling WL, Boothe AD, Ritchie AE. Characteristics of a coronavirus (strain 67N) of pigs. Am J Vet Res. 1972; 33(2): 297- 308.

21. Andries K, Pensaert MB. Immunofluorescence studies on the pathogenesis of hemagglutinating encephalomyelitis virus infection in pigs after oronasal inoculation. Am J Vet Res. 1980; 41(9): 13721378.

22. To KF, Lo AW. Exploring the pathogenesis of severe acute respiratory syndrome (SARS): the tissue distribution of the coronavirus (SARS-CoV) and its putative receptor, angiotensin-converting enzyme 2 (ACE2). J Pathol. 2004; 203: 740- 743.

23. Tang JW, To KF, Lo AW, Sung JJ, Ng HK, Chan PK. Quantitative temporal-spatial distribution of severe acute respiratory syndrome-associated coronavirus (SARS-CoV) in post-mortem tissues. J Med Virol. 2007; 79: 1245- 1253

24. George PM, Wells AU. Pirfenidone for the treatment of idiopathic pulmonary fibrosis. Expert Rev Clin Pharmacol. 2017 May;10(5):483-491. doi: 10.1080/17512433.2017.1295846. Epub 2017 Mar 7.

25. Lancaster LH, de Andrade JA et al. Pirfenidone safety and adverse event management in idiopathic pulmonary fibrosis. Eur Respir Rev. 2017 Dec 6;26(146). pii: 170057. doi: 10.1183/16000617.00572017. Print 2017 Dec 31. 
26. Chung MP, Park MS et al. Safety and Efficacy of Pirfenidone in Advanced Idiopathic Pulmonary Fibrosis: A Nationwide Post-Marketing Surveillance Study in Korean Patients.Adv Ther. 2020 Apr 15. doi: 10.1007/s12325-020-01328-8. [Epub ahead of print] 